\title{
The predictive value of systemic immune inflammation index on long-term outcomes among acute pulmonary embolism patients
}

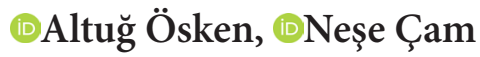 \\ Dr. Siyami Ersek Thoracic and Cardiovascular Surgery Center, Training and Research Hospital, Department of Cardiology, İstanbul, Turkey
}

Cite this article as: Ösken $\mathrm{A}$, Çam N. The predictive value of systemic immune inflammation index on long-term mortality among patients with acute pulmonary embolism. J Health Sci Med 2021; 4(5): 557-563.

\begin{abstract}
Aim: The systemic immune inflammation index (SII) has begun to be used as a useful and new marker that predicts adverse clinical outcomes in patients with malignancies and cardiovascular diseases. We performed the present study assuming that SII could provide further additional information in predicting long-term mortality in patients with acute pulmonary embolism (APE).

Material and Method: We included 504 consecutive patients followed up with APE. The study group was divided into those survivors and non-survivors. Demographic, clinical, laboratory, and echocardiographic characteristics were compared between groups.

Results: A total of 28 (5.4\%) patients died in the 30 days. Besides, during a clinical follow-up period of 29 [12-53] months, 52 patients (10.1\%) died. According to the results of the regression analysis, age [odds ratio (OR): 1.052, 95\% confidence interval (CI): 1.034-1.071; $\mathrm{p}<0.01$ ], right ventricle end-diastolic diameter basal (OR: 3.227, 95\% CI: 1.902-5.474; $\mathrm{p}<0.001$ ), left ventricular ejection fraction (OR: 0.968, 95\% CI: 0.948-0.988) and SII index (OR: 2.129, 95\% CI: 1.290-3.515) were the independent predictors of overall mortality among the study population. A receiver operating characteristic curve analysis revealed that the area under the curve values of the SII index for overall mortality was 0.703 (95\% CI: 0.629-0.777). SII with an optimal cutoff value of $1111 \times 10^{9}$ predicted the overall mortality with a sensitivity of $72 \%$ and specificity of $51 \%$.

Conclusion: The SII index, an inexpensive and easily calculable parameter, was a strong predictor of overall mortality in patients with APE.
\end{abstract}

Keywords: Inflammatory marker, pulmonary embolism, risk assessment, systemic immune-inflammation index

\section{INTRODUCTION}

Patients diagnosed with acute pulmonary embolism (APE) constitute one of the leading causes of death among cardiovascular diseases., although mortality rates have declined effectively with early diagnosis and treatment (1). Inflammation plays a crucial role in pathogenesis in venous thromboembolism (VTE) (2).

Systemic inflammation has an important role in VTE risk stratification and can be evidenced by the detection of many circulating pro-inflammatory biomarkers (3). Many clinical trials exist on hematological parameters that can predict the clinical severity and survival of APE (4-7).

Clinical composite scores, echocardiographic parameters showing right ventricular (RV) strain have been proven in studies to predict short and long-term mortality (8-13). However, there is still a need for cheap and effective predictors of mortality that can be easily calculated in a short time.

The systemic immune-inflammation (SII) index was first used in patients with malignancy and proved to be an effective predictor of mortality (14). Subsequently, studies have been published that the same index may be effective in predicting mortality in patients with acute coronary syndrome and also in predicting the clinical severity of APE (15-18). To our knowledge, there is no study investigating the relationship between the SII index and long-term mortality among patients with APE in the literature. Hence, we sought to determine 
if a simple, routinely evaluated laboratory parameter at index hospital admission is practical for long-term risk stratification in patients with APE.

\section{MATERIAL AND METHOD Study Population}

The study population included consecutive APE patients between January 2013 to December 2020 at 1 tertiary heart care center. A total of 856 patients were included in the study retrospectively from the medical records of our hospital. This study was approved by the Haydarpaşa Numune Training and Research Hospital Clinical Researches Ethics Committee (Date: 26.04.2021, Decision No: 2021/126). All procedures were carried out in accordance with the ethical rules and the principles of the Declaration of Helsinki. Patients with a clinical diagnosis and documented by imaging criteria were included. The exclusion criteria were described as the existence of active or past malignancy, hematological disorders using an immunosuppressant medication, end-stage renal or hepatic failure, collagen vascular disease, and acute or chronic infection. Therewithal, patients who died within the first 24 hours after hospitalization as a consequence of massive APE and were, therefore, could not be evaluated by echocardiographic and laboratory examination were not recruited in the present study. The final study analysis was carried out by including a total of 514 patients.

\section{Data Collection}

Clinical symptoms, predisposing factors for VTE, demographic data, laboratory, and echocardiographic examinations of the recruited study participants were obtained using the hospital database. Patients were separated into the two groups as stated in survival status. The characteristics of the collected data were compared between the groups.

Pulmonary angiographic imaging with computed tomography (CTPA) was carried out on the whole study population with basal normal creatinine values to establish the diagnosis of APE. In cases where imaging with CTPA is not possible, the confirmation was verified by ventilation/perfusion lung scanning.

A detailed echocardiographic evaluation was performed on all patients which were included in the study during the first 24 hours after admission to evaluate their RV functions.

\section{Evaluation of Echocardiographic Parameters}

Echocardiographic analyses were done by using a device (vivid S5, GE Healthcare Systems, Chicago, Illinois, USA). The LV ejection fraction (LVEF) was measured via biplane two-dimensional Simpson's method. The maximum velocity of tricuspid regurgitation (TRVmax) was calculated from the apical four-chamber (A4C) view. The pressure of the right atrium (RA) was estimated from the inferior vena cava (IVC) thickness and in line with whether or not the IVC collapsed with respiration. The estimated pulmonary arterial systolic pressure (PASP) was measured as stated by the following formula: $4 \times \mathrm{TR}-\mathrm{Vmax} 2+$ estimated RA pressure. The end-diastolic diameters of RV (RV-EDDs) were measured from the RV-focused $\mathrm{A} 4 \mathrm{C}$ at the basal segment.

\section{Definitions and Study Outcomes}

The SII index was calculated as total peripheral platelet counts $(\mathrm{P}) \times$ neutrophil-to-lymphocyte ratio $(\mathrm{N} / \mathrm{L})$ $(\mathrm{SII}=\mathrm{P} \times \mathrm{N} / \mathrm{L}$ ratio) (14). A Pulmonary Embolism Severity Index (PESI) score calculation was performed for each patient by assessing 11 clinical variables, comprising demographics (age and sex), comorbidities (chronic lung disease, cancer, heart failure), and vital functions (systolic blood pressure, heart rate, respiratory rate, body temperature, arterial oxygen saturation and altered mental status) (8).

Diagnosis of deep vein thrombosis was confirmed with lower extremity Doppler venous ultrasonography. Hypertension (HT) was described as the current use of antihypertensive medication or initial blood pressure above 140/90 $\mathrm{mmHg}$; diabetes mellitus (DM) was described as the use of anti-diabetic treatment or fasting glucose levels of $>126 \mathrm{mg} / \mathrm{dL}$ and hyperlipidemia was described as total serum cholesterol levels of $>240 \mathrm{mg} /$ dL. Current smoking status was also defined.

The glomerular filtration rate (GFR) was computed using the Cockgraft- Gault equation (19).

Long-term mortality data were collected from the patient's medical records or domestic social security institution database. In cases where it is not possible to access this data, the current survival status of patients was verified by a phone conversation with the patient's family. The main outcome of the study was the occurrence of mortality at a 30-day period or long-term follow-up which was defined as all deaths from any cause.

\section{Statistical Analysis}

Statistical analyses were conducted using the Statistical Package for the Social Sciences version 22.0 (IBM Corporation, Armonk, NY, USA). Continuous variables are given as mean \pm standard deviation or the median (interquartile range) values. Categorical variables are given as number (percentage) values. The Shapiro- 
Wilk test was applied to assess normality. Normally distributed variables were compared between groups using an independent-samples t-test. Variables that do not fit the normal distribution were compared with the Mann-Whitney U test. Evaluation of categorical variables was done using the chi-squared or Fisher's exact test. Cox-regression analysis was performed to estimate predictive markers of long-term all-cause mortality. Receiver operating characteristic (ROC) curves were constituted to examine the ability of the SII index to predict 30-day, long-term, and overall mortality rates. The long-term survival status was established by the Kaplan-Meier method, and the logrank test was used to assess differences between the two groups. A probability value of $\mathrm{p}<0.05$ was considered to be statistically significant.

\section{RESULTS}

The study population included 514 patients, who were stratified according to their survival status. The mean age of the participants was $61.5 \pm 16.1$. A total of $80(15.6 \%)$ non-survivors and $434(86.4 \%)$ survivors with APE were compared. Clinical, demographic characteristics, predisposing factors, echocardiographic parameters, and laboratory findings of the study population are provided in Table 1,2,3. History of DM and heart failure, the altered states of consciousness, fibrinolytic treatment were more prevalent among non-survivors. Age, PESI score, d-dimer levels, white blood cell, neutrophil, platelet count, and SII index were higher and GFR was lower among non-survivors. In terms of echocardiographic characteristics; RV-EDD and RVSP were significantly higher among non-survivors while those in survivors have higher LVEF.

\section{Table 1. Baseline demographic and clinical characteristics regarding the patient groups.}

\begin{tabular}{|c|c|c|c|c|}
\hline & $\begin{array}{c}\text { Study population } \\
(\mathrm{n}=514)\end{array}$ & $\begin{array}{c}\text { Non-survivors } \\
(\mathbf{n}=\mathbf{8 0})\end{array}$ & $\begin{array}{c}\text { Survivors } \\
(\mathrm{n}=434)\end{array}$ & $\mathbf{P}$ \\
\hline Age, $y$, mean & $61.5 \pm 16.1$ & $71.2 \pm 14.1$ & $59.7 \pm 15.8$ & $<0.001^{\mathrm{t}}$ \\
\hline Gender, female & $282(54.9)$ & $46(57.5)$ & $236(45.9)$ & $0.606^{x 2}$ \\
\hline Hypertension & $294(57.2)$ & $53(66.3)$ & $241(55.5)$ & $0.075^{x^{2}}$ \\
\hline Diabetes mellitus & $145(28.2)$ & $30(37.5)$ & $115(26.5)$ & $0.044^{\mathrm{x} 2}$ \\
\hline Hyperlipidemia & $119(23.2)$ & $20(25)$ & $99(22.8)$ & $0.670^{x^{2}}$ \\
\hline Smoking & $212(41.2)$ & $28(35)$ & $184(42.4)$ & $0.217^{x^{2}}$ \\
\hline Previous heart failure & $62(12.1)$ & $19(23.8)$ & $43(9.9)$ & $<0.001^{\mathrm{x} 2}$ \\
\hline Previous chronic kidney disease & $47(9.1)$ & $7(8.8)$ & $40(9.2)$ & $0.894^{x^{2}}$ \\
\hline Previous chronic obstructive pulmonary disease & $81(15.8)$ & $15(18.8)$ & $66(15.2)$ & $0.424^{x^{2}}$ \\
\hline Previous coronary artery disease & $105(20.4)$ & $17(21.3)$ & $88(20.3)$ & $0.843^{\mathrm{x}^{2}}$ \\
\hline Blood pressure (systolic) & $124 \pm 21.7$ & $119.5 \pm 22.9$ & $126 \pm 22$ & $0.103^{\mathrm{t}}$ \\
\hline Heart rate (bpm) & $100.8 \pm 18.6$ & $107.4 \pm 20.1$ & $98.2 \pm 18$ & $0.008^{t}$ \\
\hline PESI score & $99.4 \pm 36.7$ & $123 \pm 42.9$ & $95.1 \pm 33.7$ & $<0.001^{\mathrm{t}}$ \\
\hline Day of hospital stay & $8.2 \pm 5.4$ & $7.1 \pm 5.6$ & $8.4 \pm 5.4$ & $0.05^{\mathrm{t}}$ \\
\hline Main pulmonary artery involvement & $167(32.5)$ & $42(52.5)$ & $125(28.8)$ & $0.193^{x^{2}}$ \\
\hline Fibrinolytic therapy & $132(25.7)$ & $32(40)$ & $90(20.7)$ & $<0.001^{\mathrm{x} 2}$ \\
\hline Follow-up (months) & $29(12-53)$ & $9(0-32)$ & $35(18-57)$ & - \\
\hline
\end{tabular}

Table 2. Comparison of symptoms and predisposing factors regarding the patient groups.

\begin{tabular}{|c|c|c|c|c|}
\hline & $\begin{array}{c}\text { Study population } \\
(\mathrm{n}=514)\end{array}$ & $\begin{array}{c}\text { Non-survivors } \\
(\mathbf{n}=\mathbf{8 0})\end{array}$ & $\begin{array}{c}\text { Survivors } \\
(\mathrm{n}=434)\end{array}$ & $\mathbf{P}$ \\
\hline \multicolumn{5}{|l|}{ Predisposing factors } \\
\hline Immobility for more than 3 days & $68(13.2)$ & $15(18.8)$ & $53(12.2)$ & 0.113 \\
\hline Surgery in the last 1 month & $32(6.2)$ & $7(8.8)$ & $25(5.8)$ & 0.309 \\
\hline Trauma within 2 months & $22(4.3)$ & $1(1.3)$ & $21(4.8)$ & 0.145 \\
\hline Prior DVT/PE & $31(6)$ & $9(11.2)$ & $22(5.1)$ & 0.125 \\
\hline Concomitant DVT & $191(37.2)$ & $29(36.3)$ & $162(37.3)$ & 0.855 \\
\hline \multicolumn{5}{|l|}{ Symptoms } \\
\hline Chest pain & $98(19.1)$ & $10(12.5)$ & $88(20.3)$ & 0.104 \\
\hline Dyspnea & $439(85.4)$ & $71(88.8)$ & $368(84.8)$ & 0.357 \\
\hline Syncope & $114(22.2)$ & $15(18.8)$ & $99(22.8)$ & 0.422 \\
\hline Altered status of consciousness & $23(4.5)$ & $7(8.8)$ & $16(3.7)$ & 0.044 \\
\hline Pain in the lower extremity & $202(39.3)$ & $24(30)$ & $178(41)$ & 0.064 \\
\hline
\end{tabular}




\begin{tabular}{|c|c|c|c|c|}
\hline & $\begin{array}{c}\text { Study population } \\
(\mathrm{n}=514)\end{array}$ & $\begin{array}{c}\text { Non-survivors } \\
(\mathbf{n}=\mathbf{8 0})\end{array}$ & $\begin{array}{c}\text { Survivors } \\
(\mathrm{n}=434)\end{array}$ & $\mathbf{P}$ \\
\hline \multicolumn{5}{|c|}{ Echocardiographic parameters } \\
\hline RV-EDD (A4C) (cm) & $4 \pm 0.5$ & $4.2 \pm 0.4$ & $3.9 \pm 0.5$ & $<0.001^{\mathrm{t}}$ \\
\hline RVSP (mmHg) & $50 \pm 12.5$ & $51.4 \pm 16.4$ & $49.8 \pm 11.7$ & $0.283^{\mathrm{t}}$ \\
\hline LVEF (\%) & $57.3 \pm 7.9$ & $54.1 \pm 11$ & $57.9 \pm 7$ & $0.004^{t}$ \\
\hline \multicolumn{5}{|l|}{ Laboratory parameters } \\
\hline Glucose, $\mathrm{mg} / \mathrm{dL}$ & $162 \pm 76.9$ & $184.9 \pm 93.2$ & $157.8 \pm 73$ & $0.017^{\mathrm{t}}$ \\
\hline Baseline Creatinine, mg/dL & $1.15 \pm 0.63$ & $1.19 \pm 0.4$ & $1.15 \pm 0.66$ & $0.625^{\mathrm{t}}$ \\
\hline D-dimer (ng/mL) & $2871(1522-5587)$ & $3278(1716-8716)$ & $2700(1522-5466)$ & $0.031^{\mathrm{m}}$ \\
\hline Cardiac troponin $(\mathrm{ng} / \mathrm{mL})$ & $0.33(0.09-1.19)$ & $0.32(0.04-1.35)$ & $0.33(0.09-1.18)$ & $0.605^{\mathrm{m}}$ \\
\hline NT-proBNP (pg/dL) & $318(143-577)$ & $320(97-927)$ & $318(144-529)$ & $0.986^{\mathrm{m}}$ \\
\hline GFR, $\mathrm{mL} / \mathrm{min}$ & $66.6 \pm 25.4$ & $59 \pm 23.4$ & $68 \pm 25.6$ & $0.003^{t}$ \\
\hline Hemoglobin, g/Dl & $12.7 \pm 3.1$ & $12.3 \pm 1.8$ & $12.8 \pm 3.3$ & $0.204^{\mathrm{t}}$ \\
\hline $\mathrm{WBC}, \times 10^{9} / \mathrm{L}$ & $11.9 \pm 3.9$ & $13.3 \pm 4.9$ & $11.6 \pm 3.7$ & $0.004^{t}$ \\
\hline Neutrophil, $\times 10^{9} / \mathrm{L}$ & $9.1 \pm 3.3$ & $10.5 \pm 4.2$ & $8.9 \pm 3.1$ & $<0.001^{\mathrm{t}}$ \\
\hline Lymphocyte, $\times 10^{9} / \mathrm{L}$ & $1.7 \pm 0.8$ & $1.6 \pm 1.1$ & $1.8 \pm 0.8$ & $0.085^{\mathrm{t}}$ \\
\hline Platelet, $\times 10^{9} / \mathrm{L}$ & $230.4 \pm 78.2$ & $251.4 \pm 81.9$ & $226.5 \pm 77$ & $0.009^{t}$ \\
\hline SII index & $1193(783-1769)$ & $1918(1032-3326)$ & $1131(769-1647)$ & $<0.001^{m}$ \\
\hline
\end{tabular}

While 28 (5.4\%) of the patients included in the study died in the 30 days, during a follow-up period of 29 [12-53] months, 52 patients (10.1\%) died. The overall mortality rate was $15.6 \%$.

To identify independent predictors of overall mortality, we performed a Cox regression analysis of the whole study population. The univariate analysis revealed that age, DM, HT, GFR, RV-EDD, LVEF, WBC, neutrophil, lymphocyte, platelet count, and SII index were ascertained as potential independent predictors of overall mortality among patients with APE. In the multivariate regression analysis, age [odds ratio (OR): 1.052, 95\% confidence interval (CI): 1.034-1.071; $\mathrm{p}<0.01$ ], RV-EDD (OR: 3.227, 95\% CI: 1.902 5.474; p<0.001), LVEF (OR: 0.968, 95\% CI: 0.948-0.988) and SII index (OR: 2.129, 95\% CI: 1.290-3.515) continued to exist being the independent overall mortality predictors among the study population (Table 4).
The SII index significantly predicted the rates of 30-day and long-term mortality as well as the rate of overall mortality. ROC analysis revealed that the provided area under the curve (AUC) values of the SII index for 30-day mortality, long-term, and overall mortality rates were 0.623 (95\% CI: $0.504-0.742$ ), 0.724 (95\% CI: $0.634-$ 0.813 ) and 0.703 (95\% CI: $0.629-0.777)$, respectively (Figures 1a-c).

SII index $>1111 x 109$ cut-off value predicted overall mortality with a sensitivity of $72 \%$ and a specificity of $51 \%$. Additionally, the Kaplan-Meier curve for patients with SII index over the cut-off values had a significantly higher risk for overall mortality $(90.9 \%$ vs $79.1 \%$, respectively, log-rank test $\mathrm{p}$ value $<0.001$ ) (Figure 2).

\begin{tabular}{|c|c|c|c|c|c|}
\hline Univariate analysis & $\mathbf{P}$ & OR (95\% CI) & Multivariate analysis & $\mathbf{P}$ & OR (95\% CI) \\
\hline Age & $<0.001$ & $1.052(1.035-1.070)$ & Age & $<0.001$ & $1.052(1.034-1.071)$ \\
\hline Gender (Female) & 0.535 & $0.869(0.558-1.354)$ & & & \\
\hline Diabetes mellitus & 0.020 & $1.709(1.086-2.689)$ & & & \\
\hline Hypertension & 0.037 & $1.640(1.030-2.610)$ & & & \\
\hline Smoking & 0.531 & $0.863(0.544-1.369)$ & & & \\
\hline Fibrinolytic therapy & 0.109 & $0.693(0.443-1.085)$ & & & \\
\hline $\mathrm{GFR}, \mathrm{mL} / \mathrm{min}$ & 0.011 & $0.988(0.980-0.997)$ & & & \\
\hline RV-EDD (A4C) $(\mathrm{cm})$ & $<0.001$ & $3.601(2.162-5.999)$ & RV-EDD (A4C) (cm) & $<0.001$ & $3.227(1.902-5.474)$ \\
\hline RVSP (mmHg) & 0.513 & $1.006(0.989-1.023)$ & & & \\
\hline LVEF (\%) & $<0.001$ & $0.964(0.945-0.983)$ & LVEF (\%) & 0.002 & $0.968(0.948-0.988)$ \\
\hline WBC, $\times 10^{9} / \mathrm{L}$ & 0.001 & $1.081(1.032-1.132)$ & & & \\
\hline Neutrophil, $\times 10^{9} / \mathrm{L}^{*}$ & $<0.001^{\star}$ & $1.119(1.057-1.185)$ & & & \\
\hline Lymphocyte, $\times 10^{9} / \mathrm{L}^{*}$ & $0.033^{*}$ & $0.722(0.534-0.975)$ & & & \\
\hline Platelet, $\times 10^{9} / \mathrm{L}^{*}$ & $0.028^{*}$ & $1.002(1.000-1.005)$ & & & \\
\hline SII index $>1111$ & $<0.001$ & $2.398(1.457-3.947)$ & SII index $>1111$ & 0.003 & $2.129(1.290-3.515)$ \\
\hline
\end{tabular}




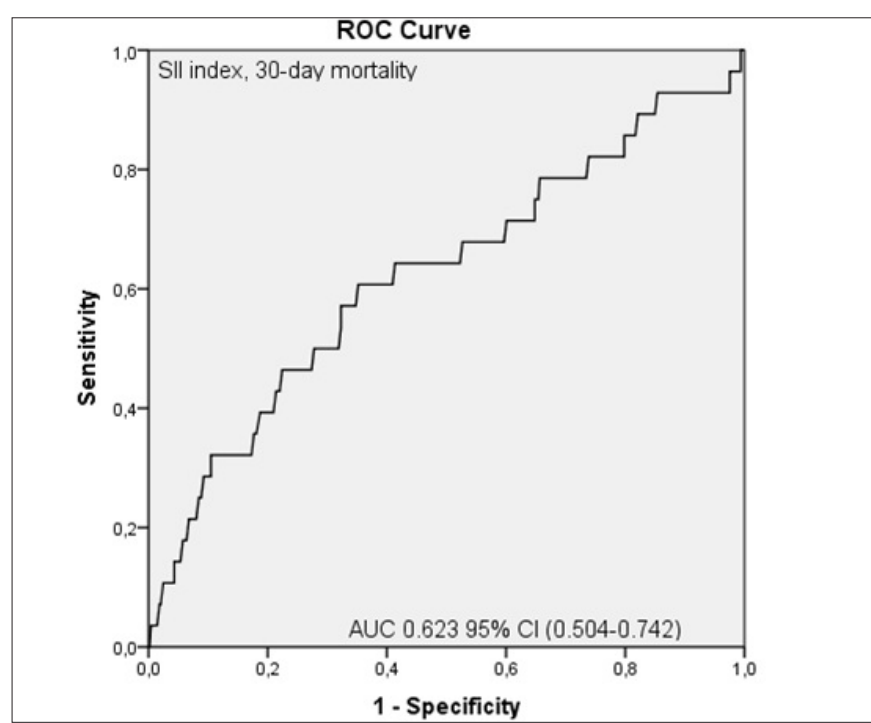

Figure 1a. Receiver operating characteristic (ROC) curves of SII index and 30-day mortality

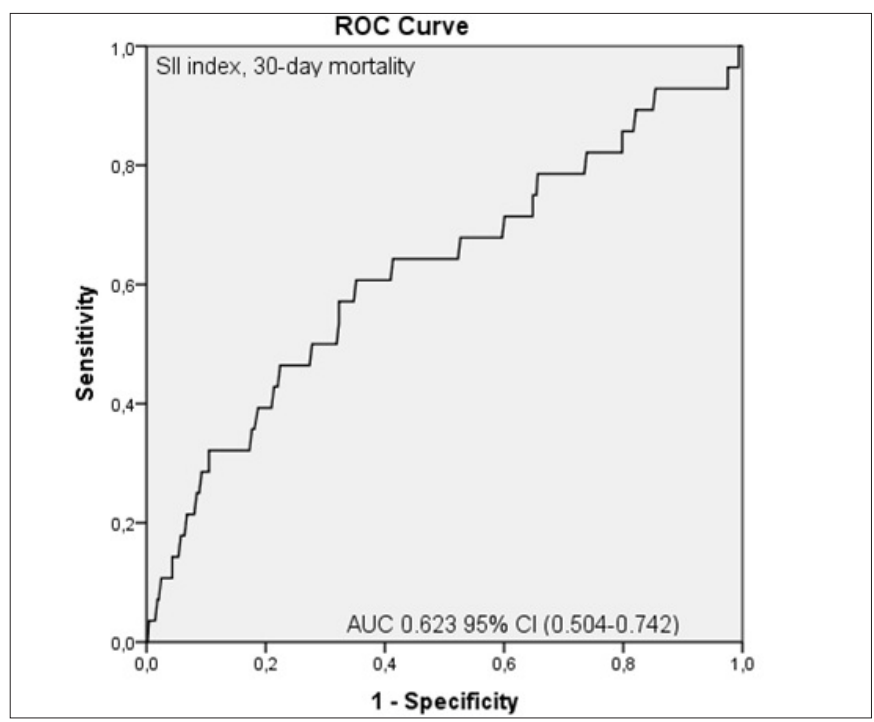

Figure 1b. Receiver operating characteristic (ROC) curves of SII index and long-term mortality

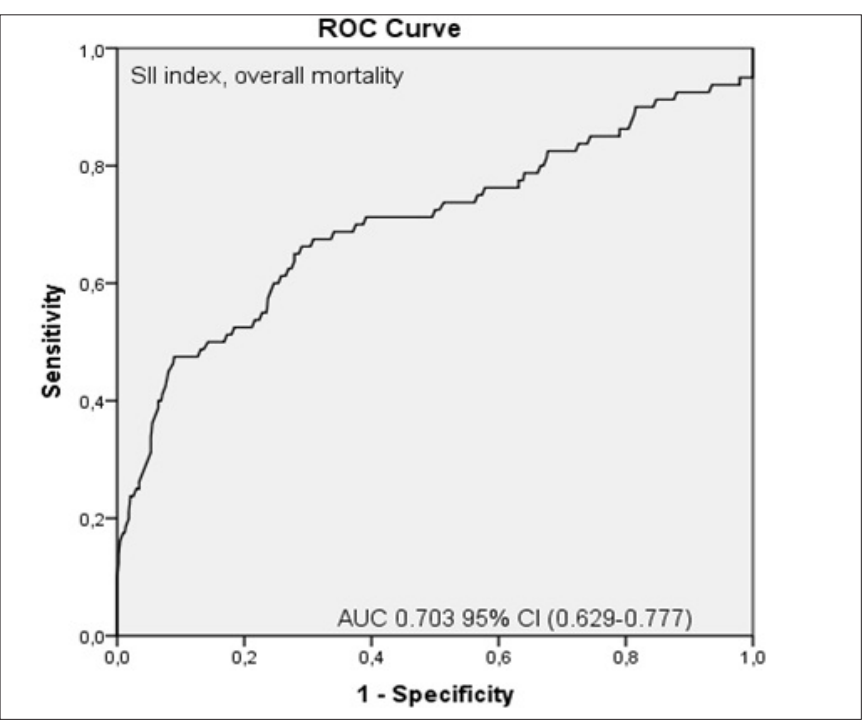

Figure 1c. Receiver operating characteristic (ROC) curves of SII index and overall mortality

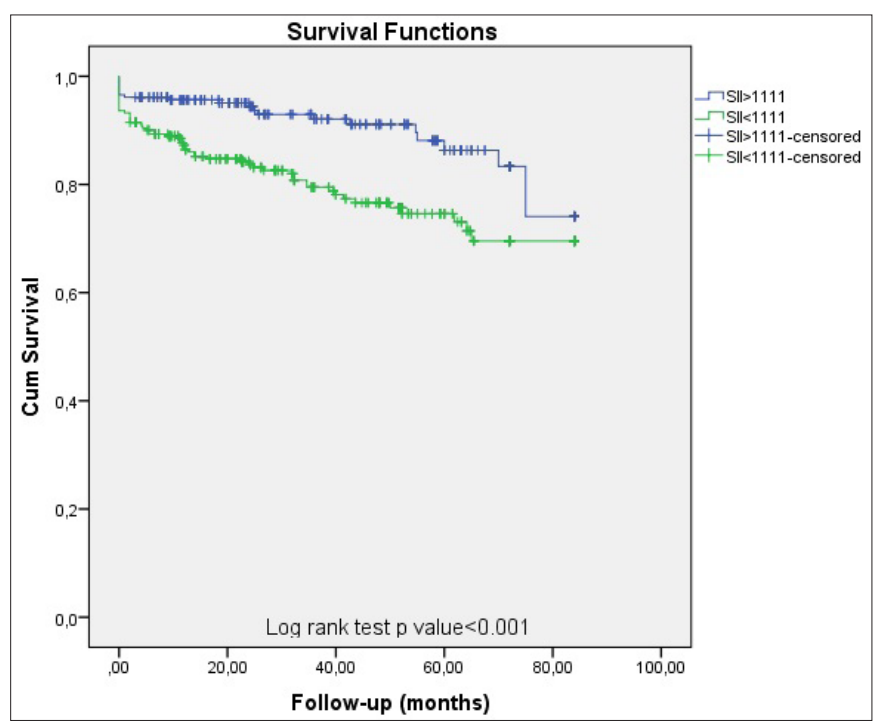

Figure 2. Kaplan-Meier survival curve for overall survival in study population stratified by SII index cut-off values.

\section{DISCUSSION}

The main finding of the present study was that a higher SII index boasts good accuracy for predicting all-cause short and long-term mortality in patients with APE. We hypothesized that patients with a higher SII index might experience a more unfavoured prognosis.

An increase in the release of pro-coagulant and proinflammatory substances originating from endothelial cells, leukocytes, and platelets was observed in the clinical course of APE (20). Platelet activation and neutrophilic recruitment resulting from the acute inflammatory response were associated with unfavorable prognosis and mortality in APE patients (21). SII is a simple, cheap, and objective index especially studied in patients with malignancy that is thought to reflect the equilibration between the immune and inflammatory responses of the host better than other systemic markers (22). Higher SII generally suggests a reinforced inflammatory and inadequate immune response. It is not known the mechanism for the increased SII index after the onset of APE; however, acute right ventricular overload, circulatory collapse, and increased inflammatory response are thought to be potential causes in the setting of APE.

Many studies are arguing that platelet to lymphocyte ratio and neutrophil to lymphocyte ratio may be short-long term mortality predictors in patients with APE $(4-6,23)$. Neutrophils could play an important role in modulating systemic inflammation and may be used as an indicator of increased inflammatory response. However, in cases of increased apoptosis triggered by stress, together with increased corticosteroid levels, a marked decrease in lymphocyte counts can be observed. In addition, high platelet counts can lead to increased platelet activation and the release of inflammatory mediators, therefore susceptibility to thrombosis occurs (24). 
Since the SII index is considered to be an important inflammatory marker, it has been argued that it may be a prognosticator of long-term deaths and adverse events in many cardiovascular diseases. Huang et al. (15) showed that the SII index strongly predicted clinical outcomes, especially in selected elderly patients with acute coronary syndromes. In a study conducted with a large cohort, Yang et al. (16) found that higher SII scores predicted the development of cardiac events after percutaneous coronary intervention. In a recent study, Candemir et al. (17) demonstrated a significant relationship between the severity of coronary artery stenosis calculated using angiographic parameters and the SII index. Furthermore, Gok et al. (18) argued that SII levels were significantly greater in massive APE patients, and this significance progressively increased from nonmassive to massive. However, only in-hospital mortality was evaluated in this study. No studies exist in the literature showing that SII may be a predictor of mortality in long-term follow-up in patients with APE. Thus, we can conclude that this is the initial study to show the long-term prognostic value of the SII index in APE.

We also showed that, in addition to the SII index; age, RV-EDD and LVEF predicted the occurrence of overall mortality entire study population. Age is one of the most important factors affecting the severity of APE and the frequency of adverse events. A significant increase in VTE mortality is observed in elderly patients (25). Conflicting results exist in terms of mortality in patients with RV dysfunction and dilatation on echocardiography $(26,27)$. Since echocardiography is a subjective evaluation, it has been shown that RV diameters and RV/LV ratios measured by computed tomography are determinants in terms of mortality (28). Both the incidence of APE and the frequency of mortality increase significantly in heart failure patients with low $\operatorname{LVEF}(29,30)$.

\section{Limitations}

Some limitations exist to be considered in our study. First, this was intended as a single-center retrospective cohort study. Second, the SII index was evaluated only at admission; it would be remarkable to assess whether the SII index alters over time and to determine whether the patient's baseline value differences evolve as they suffer the undesirable event. Prospective clinical followup trials are needed to generalize these findings to larger patient populations.

\section{CONCLUSION}

This study indicates that the baseline SII index revealed significant predictive value for overall mortality on longterm follow-up in patients with APE. This parameter is easy to calculate and cheap; so, it might be used to distinguish high-risk patients with the worst outcomes and to provide a unique point of view in the treatment, assessment, and prognosis of patients with APE.

\section{ETHICAL DECLARATIONS}

Ethics Committee Approval: This study was approved by the Haydarpaşa Numune Training and Research Hospital Clinical Researches Ethics Committee (Date: 26.04.2021, Decision No: 2021/126).

Informed Consent: Because the study was designed retrospectively, no written informed consent form was obtained from patients.

Referee Evaluation Process: Externally peer-reviewed.

Conflict of Interest Statement: The authors declare that they have no conflicts of interest to disclose.

Financial Disclosure: The authors declared that this study has received no financial support.

Author Contributions: All of the authors declare that they have all participated in the design, execution, and analysis of the paper, and that they have approved the final version.

\section{REFERENCES}

1. Martin KA, Molsberry R, Cuttica MJ, et al. Time Trends in Pulmonary Embolism Mortality Rates in the United States, 1999 to 2018. J Am Heart Assoc 2020; 9: e016784.

2. Vazquez-Garza E, Jerjes-Sanchez C, Navarrete A, Joya-Harrison J, Rodriguez D. Venous thromboembolism: thrombosis, inflammation, and immunothrombosis for clinicians. J Thromb Thrombolysis 2017; 44: 377-85.

3. Branchford BR, Carpenter SL. The role of inflammation in venous thromboembolism. Front Pediatr 2018; 6: 142.

4. Wang Q, Ma J, Jiang Z, et al. Prognostic value of neutrophilto-lymphocyte ratio and platelet-to-lymphocyte ratio in acute pulmonary embolism: a systematic review and meta-analysis. Int Angiol 2018; 37: 4-11.

5. Karataş MB, İpek G, Onuk T, et al. Assessment of prognostic value of neutrophil to lymphocyte ratio and platelet to lymphocyte ratio in patients with pulmonary embolism. Acta Cardiol Sin 2016; 32: 313-20.

6. Phan T, Brailovsky Y, Fareed J, et al. Neutrophil-to-lymphocyte and platelet-to-lymphocyte ratios predict all-cause mortality in acute pulmonary embolism. Clin Appl Thromb Hemost 2020; 26: 1076029619900549.

7. Araz O, Yilmazel Ucar E, Yalcin A, et al. Predictive value of serum Hs-CRP levels for outcomes of pulmonary embolism. Clin Respir J 2016; 10: 163-7.

8. Donzé J, Le Gal G, Fine MJ, et al. Prospective validation of the pulmonary embolism severity index. A clinical prognostic model for pulmonary embolism. Thromb Haemost 2008; 100: 943-8.

9. Aujesky D, Obrosky DS, Stone RA, et al. Derivation and validation of a prognostic model for pulmonary embolism. Am J Respir Crit Care Med 2005; 172: 1041-6.

10. Vedovati MC, Cimini LA, Pierpaoli L, et al. Prognostic value of respiratory index in haemodynamically stable patients with acute pulmonary embolism: The Respiratory Index model study. Eur Heart J Acute Cardiovasc Care 2020; 9: 286-92. 
11. Burgos LM, Scatularo CE, Cigalini IM, et al. The addition of echocardiographic parameters to PESI risk score improves mortality prediction in patients with acute pulmonary embolism: PESI-Echo score. Eur Heart J Acute Cardiovasc Care 2020: zuaa007.

12. Lyhne MD, Kabrhel C, Giordano N, et al. The echocardiographic ratio tricuspid annular plane systolic excursion/pulmonary arterial systolic pressure predicts short-term adverse outcomes in acute pulmonary embolism. Eur Heart J Cardiovasc Imaging 2021; 22: 285-94.

13. Kucher N, Rossi E, De Rosa M, et al. Prognostic role of echocardiography among patients with acute pulmonary embolism and a systolic arterial pressure of $90 \mathrm{mmHg}$ or higher. Arch Intern Med 2005; 165: 1777-81.

14. Hu B, Yang XR, Xu Y, et al. Systemic immune-inflammation index predicts prognosis of patients after curative resection for hepatocellular carcinoma. Clin Cancer Res 2014; 20: 6212-22.

15. Huang J, Zhang Q, Wang R, et al. Systemic immune-inflammatory index predicts clinical outcomes for elderly patients with acute myocardial infarction receiving percutaneous coronary intervention. Med Sci Monit 2019; 25: 9690-701.

16. Yang YL, Wu CH, Hsu PF, et al. Systemic immune-inflammation index (SII) predicted clinical outcome in patients with coronary artery disease. Eur J Clin Invest 2020; 50: e13230.

17.Candemir M, Kiziltunç E, Nurkoç S, et al. Relationship between systemic immune-inflammation index (SII) and the severity of stable coronary artery disease. Angiology 2021: 3319720987743.

18. Gok M, Kurtul A. A novel marker for predicting severity of acute pulmonary embolism: systemic immune-inflammation index. Scand Cardiovasc J 2021; 55: 91-6.

19. Cockcroft DW, Gault MH. Prediction of creatinine clearance from serum creatinine. Nephron 1976; 16: 31-41.

20.Galeano-Valle F, Ordieres-Ortega L, Oblitas CM, et al. Inflammatory Biomarkers in the Short-Term Prognosis of Venous Thromboembolism: A Narrative Review. Int J Mol Sci 2021; 22 2627.

21.Jo JY, Lee MY, Lee JW, Rho BH, Choi WI. Leukocytes and systemic inflammatory response syndrome as prognostic factors in pulmonary embolism patients. BMC Pulm Med 2013; 13: 74.

22.Li C, Tian W, Zhao F, et al. Systemic immune-inflammation index, SII, for prognosis of elderly patients with newly diagnosed tumors. Oncotarget 2018; 9: 35293-9.

23. Duman D, Sonkaya E, Ylldırım E, et al. Association of Inflammatory Markers with Mortality in Patients Hospitalized with Non-massive Pulmonary Embolism. Turk Thorac J 2021; 22: 24-30.

24.Davì G, Patrono C. Platelet activation and atherothrombosis. N Engl J Med 2007; 357: 2482-94.

25.Spirk D, Husmann M, Hayoz D, et al. Predictors of in-hospital mortality in elderly patients with acute venous thromboembolism: the SWIss Venous ThromboEmbolism Registry (SWIVTER). Eur Heart J 2012; 33: 921-6.

26. Stein PD, Beemath A, Matta F, et al. Enlarged right ventricle without shock in acute pulmonary embolism: prognosis. Am J Med 2008; 121: 34-42.

27. Coutance G, Cauderlier E, Ehtisham J, et al. The prognostic value of markers of right ventricular dysfunction in pulmonary embolism: a meta-analysis. Crit Care 2011; 15: R103.

28. Quiroz R, Kucher N, Schoepf UJ, et al. Right ventricular enlargement on chest computed tomography: prognostic role in acute pulmonary embolism. Circulation 2004; 109: 2401-4.

29. Dries DL, Rosenberg YD, Waclawiw MA, et al. Ejection fraction and risk of thromboembolic events in patients with systolic dysfunction and sinus rhythm: evidence for gender differences in the studies of left ventricular dysfunction trials. J Am Coll Cardiol 1997; 29: 1074-80.
30.Slobodan O, Boris D, Bojana S, et al. Predictive value of heart failure with reduced versus preserved ejection fraction for outcome in pulmonary embolism. ESC Heart Fail 2020; 7: 406170 